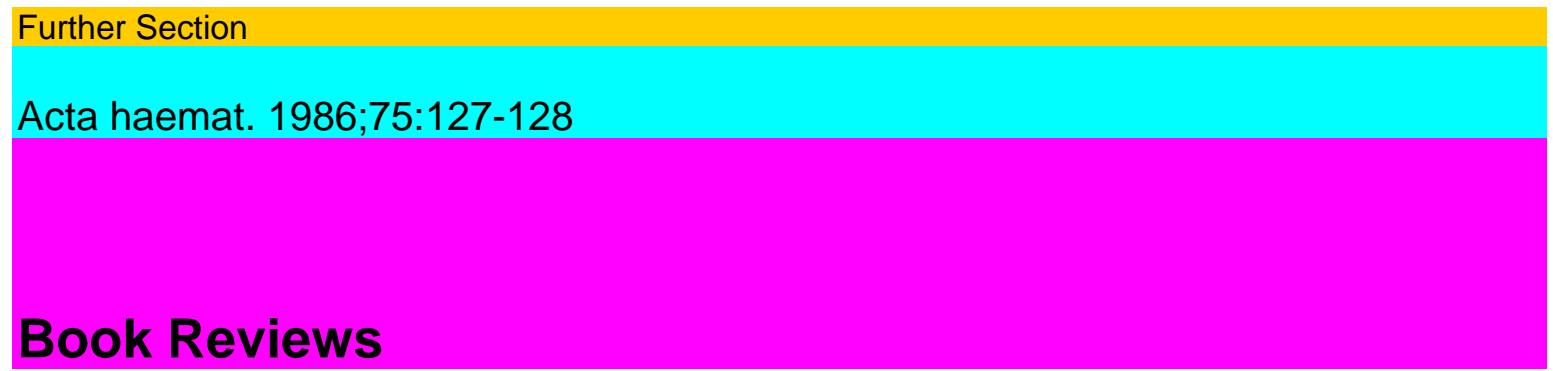

\title{
J.J. Sotto
}

Non-Hodgkin's Lymphomas: New Techniques and Treatments

Karger, Basel 1985

VIII + 232 pp.; SFr. 162.-; DM 194.-; US\$90.00

ISBN 3-8055-4064-7

This 232-page publication consists of 33 papers presented at the Cancer Research Workshop for Non-Hodgkin's Lymphomas at Grenoble in 1984. The first goal of the workshop was to take stock of the current knowledge in this field. This has been achieved in this book through the individual presentations and their references. The subject material has been well indexed by the authors, and specific topics are easily found.

The problem of finding a universally acceptable and reproducible histologic classification with simultaneous clinical and prognostic significance is evident throughout. It is found in the 2 chapters dealing with epidemiology and the 4 chapters concerning morphology. Here, the 2 papers on cytology offer some hope for improvement. The results seem to be fairly well reproducible and are of prognostic significance for the high- and low-grade malignant types. As with the Working Formulation, the cytological intermediate group has a poor prognostic correlation, but this group is relatively small. Morphometric studies are of little value for Tcell tumors but may identify true histiocytic forms. Also the biologic methods of classification have as yet failed to provide a universal solution. New improved techniques for immunohistologic studies on paraffin sections may increase the identification of monoclonal B-cell lines. Cytochemical markers, although having consistent patterns for certain nonHodkin lymphomas and leukemias, can only be considered as a complementary aid in diagnosis. Systemic markers are totally type-nonspecific and fail completely in minimal or residual disease situations. Cytogenetics have revealed a large number of abnormalities, especially in the $14 \mathrm{q}$ region. Specific changes, however, can only be claimed for Burkitt's lymphoma with its 8-14 translocation. Of interest is the paper on cells associated with the malignant clones. The decrease or absence of dendritic reticulum cells and natural killer cells in the high-grade lymphomas may be of prognostic significance.

A total of 21 papers are devoted to treatment, 9 of which consider new methods. The role of surgery is well handled, whereby the indication for and use of larger staging procedures remain controversial. New drugs are adequately reviewed. Unfortunately, the only paper on total nodal irradiation reports only on a small number of selected chronic lymphatic leukemia cases with short follow-up. Antileukocyte monoclonal antibodies are very well discussed, especially as to the reasons for failure and possibilities to correct them as well as possible new uses for these substances. The results of autologous marrow transplantation are presented for a very he-terologous patient and treatment population. The indication and techniques available for in vitro marrow purge are well presented.

The necessity for intensive treatment with CNS prophylaxis versus minimal or no treatment at all, depending upon stage and histology is well handled in the remaining papers on 
indications for and results of treatment. Special attention to mediastinal and gastrointestinal tumors is provided. The difference between low-grade malignancies with a slow but continuous fall in survival and the high-grade malignant tumors with a rapid fall which plateaus, usually at 2 years, is confirmed in several reports.

Although a few papers have obviously suffered to some extent

in the English translation, this book does provide a good summary

of the current concepts and problems in the diagnosis and treat

ment of non-Hodgkin's lymphomas. R. Sonntag, Bern

M.L. Thakur

Radiolabeled Cellular Blood Elements

Pathophysiology, Techniques, and

Scintigraphic Applications

NATO ASI Series, Series A: Life Sciences, vol. 88

Plenum Press, New York 1985

$\mathrm{X}+434$ pp.; US\$ 69.50, bound

ISBN 0-306-41935-1

This book, which appears in the 'NATO ASI series', is edited and authored by a number of the most prominent writers on radio-labeling blood cells. It is based on the contributions of guest lecturers to a meeting on radiolabeled cellular blood elements, held in Maratea, Italy, in 1982. As Dr. Thakur mentions in his preface, this book is a monograph outlining problems and potential solutions, with a critical look at a variety of techniques used and applied in vivo. It contains, therefore, chapters on basic cell physiology and critical reviews of years of data and experiences generated in the preparation and use of radiolabeled blood cells. With respect to platelet and leukocyte studies, Dr. Thakur has succeeded admirably in this goal. Research done on erythrocytes, however, is under-represented. Though the book mentions numerous items briefly, it does not have an encyclopedic character.

Clarity and practicality seem to be the keys to the basic science and clinical sections of the book, which consists of 434 pages organized into several groups of chapters. The first part deals with pathophysiology, biochemistry and kinetics of platelets and leukocytes and the evaluation of some animal models used. Although Indium-11 1 is most commonly used for tagging, other labeling methods are critically discussed. Technical prescriptions are beautifully presented, what makes their use in routine practice easy. Dosimetry and instrumentation are adequately covered and references for these chapters are also excellent. In a second group of chapters, clinical applications are discussed in detail. Radiolabeled platelets have been widely used in thromboembolic research, and many of the most recent results are analyzed in a critical manner. Many years of experience with platelet scintigraphy in myocardial infarction, embolism, vessel diseases, management of renal transplant and experimental bypass grafting are reported and documented by illustrative cases. The section dealing with the clinical application of Indium-111-labeled leukocytes for the diagnosis of infection is particularly interesting. Although much progress was made in harvesting and labeling neutrophil leukocytes, which lead to high sensitivity and specificity rates of the leukocyte scintigraphy, all methods are safe only in well trained hands. Therefore, new trends for the in vivo labeling blood cells are discussed e.g. the use of less toxic tracers or monoclonal antibodies. In a short part, an interesting review of the clinical use of radiolabeled red blood cells is given. Due 
128

Book Reviews

to lack of space, many important applications, eg. in cardiology, are not mentioned. However, references are excellent and all important studies of the recent years are noted.

In summary, the book serves its stated purpose of providing a resource for both the clinical imager and the hematologist. The set up of the book is adequate, and the photographic reproductions are quite good. Its length, references and moderate price make the book attractive, but it is above all the clear content and organization of the text which provide the greatest appeal of this book.

J.T. Locher, Aarau

Annabelle S.J. Baughan, Andrew S.B. Hughes, et al. Manual of Haematology

Churchill Livingstone, Edinburgh 1985 X +274 pp.; E8.95 ISBN 0-443-02564-9

The compact text is a helpful guide for staff members in internal medicine and for internists in private practice. Each chapter con tains an appropriate program for investigations considering the clinical situation and cost. Excellent brief guidelines are given for

the treatment. H.R. Marti, Aarau

RolfNeth, Robert C. Gallo,

Melvyn F. Greaves, Gritta Janka (eds)

Modern Trends in Human Leukemia VI

New Results in Clinical and Biological Research Including Pediatric Oncology, Haematology and Blood Transfusion Springer, Berlin 1985 XXXII + 521 pp.; DM 198.-ISBN 3-54015329-2

The book contains reports presented at the Wilsede Meeting or at the Wilsede Joint Meeting on Pediatric Oncology in June 1984. The first section deals mainly with clinical aspects of leukemia and lymphoma, e.g. chemotherapeutic approaches, allogeneic and au-tologous bone marrow transplantation, elimination of $\mathrm{T}$ cells in donor marrow in the allogeneic and elimination of residual leukemic cells in the autologous situation, supportive care, characterization of leukemic cells with immunological markers, chromosomal abnormalities and oncogene activation. The second part is about mechanisms of malignant transformation. First, biological aspects of cellular and viral oncogene expression and its regulation are discussed, followed by a series of articles on proliferation and differentiation of leukemic cells and the role of growth factors in these basic processes. Immunological aspects in malignancy are presented in the third section of the book.

The great number of short articles mediates a good general view on what is actually going on in the field of clinical and biological leukemia research. For the reader who wants to get a more extensive information about a certain aspect of leukemia research, each article contains a pertinent list of references. The book can be highly recommended to clinicians caring for patients with leukemia as well as to any basic researcher in the wide field of leukemia.

A. Hirt, Bern

J.M. Goldman, D.G. Harnden

Genetic Rearrangements in Leukaemia and Lymphoma

Leukaemia and Lymphoma Research, vol. 2 Churchill Livingstone, Edinburgh 1986179 pp.; E40.-ISBN 0-443-03102-9 
Here is an up-dated and informative review of the genetic aspects and the molecular biology of haematological neoplasias. The first chapter surveys inherited predispositions to malignant ne-oplasia, chromosome aberrations in leukaemia and lymphoma, and activation or induction of specific genes involved in the proliferation of malignant cells. For a concise summary of chromosome rearrangements the reader is referred to chapter 2 where various examples of chromosome changes in both acute and chronic leukaemia as well as in malignant lymphomas are listed. Ph'+ chronic myelogenous leukaemia and Burkitt's lymphoma (BL) are more extensively discussed in other sections. BL has undeniably become a classical example of a tumour in which mechanisms of malignant neoplastic proliferation have been studied at a molecular level. The involvement of the c-myc oncogene in chromosomal translocations in this particular lymphoma is dealt with by 4 different authors. There is an outstanding discussion of rearrangements of human antibody and $\mathrm{T}$ cell receptor genes, illustrating the state of the field both in normal and abnormal immuno-competent cells. The references provided are extensive and include original articles as well as reviews. In their preface, the editors rightly state that such a volume comes at a timely moment. It will certainly appeal to research workers, to haematologists and clinical oncologists. Throughout the text, it offers a clear and modern insight into molecular biology of haematological neoplasias. However, it may be anticipated that, given the pace of the developments in this highly competitive field, the editors will soon have to start with a new edition. The book can be recommended as an excellent introduction into a fascinating aspect of current cancer research. M.F. Fey, Bern

Ralph Snyderman

Regulation of Leukocyte Function

Contemporary Topics in Immunobiology, vol. 14 Plenum Publ., New York 1984 XIX+ 410 pp.; US \$55.00 ISBN 0-306-41585-2

This volume presents in 11 chapters different aspects of leukocyte functions. The first three of them discuss the granulocyte receptors, causing chemotactic responses. Further chapters deal with the interactions with complement factors, the production of lipox-ygenase, structure and modulation of Fc-receptors, leukocyte de-granulation, exocytosis, and finally antimicrobial reactions. The last chapter delivers detailed description of clinical malignancies, caused by disorder of leukocyte functions.

This book represents a fundamental and up-to-date work; the authors of the different chapters are highly qualified. The references have been newly edited. This book is highly recommendable to all interested theoretical and clinical scientists. H. Braunsteiner, Innsbruck 